\title{
Distributed Kerr-lens mode locking based on spatiotemporal dissipative solitons in multimode fiber lasers
}

\author{
Vladimir L. Kalashnikov (1) and Stefan Wabnitz ${ }^{\dagger}{ }^{\dagger}$ \\ Dipartimento di Ingegneria dell'Informazione, Elettronica e Telecomunicazioni, Sapienza Università di Roma, \\ via Eudossiana 18, 00184 Rome, Italy
}

(Received 3 April 2020; revised 19 June 2020; accepted 9 July 2020; published 6 August 2020)

\begin{abstract}
We introduce a mechanism of stable spatiotemporal soliton formation in a multimode fiber laser. This is based on spatially graded dissipation, leading to distributed Kerr-lens mode locking. Our analysis involves solutions of a generalized dissipative Gross-Pitaevskii equation. This equation has a broad range of applications in nonlinear physics, including nonlinear optics, spatiotemporal pattern formation, plasma dynamics, and Bose-Einstein condensates. We demonstrate that the careful control of dissipative and nondissipative physical mechanisms results in the self-emergence of stable $(2+1)$-dimensional dissipative solitons. Achieving such a regime does not require the presence of any additional dissipative nonlinearities, such as a mode locker in a laser, or inelastic scattering in a Bose-Einstein condensate. Our method allows for stable energy (or "mass") harvesting by coherent localized structures, such as ultrashort laser pulses or Bose-Einstein condensates.
\end{abstract}

DOI: 10.1103/PhysRevA.102.023508

\section{INTRODUCTION}

The endeavor of multidimensional pattern formation [1] bridges nonlinear wave collapse and turbulence phenomena [2], leading to the formation of highly coherent structures, such as solitons in nonlinear optics (the so-called "light bullets") and liquid crystals, Bose-Einstein condensates (BEC), etc. [3-6]. These coherent and strongly localized structures could provide unprecedented energy (or mass) condensation, and lead to establish connections or analogies between microand macroscaled phenomena. The study of multidimensional solitons introduces a new branch of "mesoscopic" physics, permitting the study of a broad area of nonlinear phenomena far from the thermodynamic equilibrium. The main obstacle is that, in contrast with one-dimensional (1D) soliton solutions of integrable nonlinear wave equations, higher-dimensional structures are generally unstable. Two main approaches have been proposed for the stabilization of a multidimensional soliton, which use either (i) trapping potentials in a nondissipative system [3,4,7-10], or (ii) localized nonlinear dissipation [11-14].

Nonlinear optical systems furnish an ideal playground in this field, by providing a "metaphoric" (or "analogous") modeling, big data, and rare events analysis approach $[15,16]$. Specifically, field trapping is an inherent consequence of spatial mode formation in a laser or in a passive fiber, where nonlinear effects play a decisive role. In a graded-index (GRIN) multimode fiber (MMF), the effect of spatial mode cleaning,

\footnotetext{
*Also at Institute of Photonics, Vienna University of Technology, Gusshausstrasse 25-29, Vienna 1040, Austria; vladimir.kalashnikov@uniroma1.it

${ }^{\dagger}$ Also at Novosibirsk State University, Pirogova 1, Novosibirsk 630090, Russia; stefan.wabnitz@uniroma1.it
}

or field self-condensation in the lowest-order spatial modes induced by a nonlinear intermodal interaction was recently described [5,17-19]. As it was conjectured, spatially profiled active-ion doping could enhance this effect [20]. Also, the growth of an effective gain with power (e.g., due to nonlinear polarization rotation) could result in spatiotemporal mode locking and self-similar pulse evolution in an MMF laser $[21,22]$. As a result, dissipative nonlinearities may produce coherent, localized, and energy-scalable structures, that is, dissipative solitons (DSs) [23].

A remarkable breakthrough has been achieved by the development of ultrafast fiber and solid-state waveguide lasers, that allow for avoiding the issues of thermal effects and environmental sensitivity, while providing high gain, coverage of a broad spectral range [24,25], and extremely high ultrashort-pulse repetition rates [26]. However, the presence of optical nonlinearities, such as self-phase modulation (SPM), four-wave mixing, and stimulated Raman scattering, limit ultrashort-pulse energy harvesting. A breakthrough approach was invented by introducing solid-state Kerr-lens mode-locked (KLM) oscillators. These sources exploit the effect of loss decrease due to spatial mode squeezing through self-focusing in a nonlinear medium with an aperture [27]. The evolution of KLM technology opens the perspective for achieving distributed KLM, or DKLM [28,29]. This permits us to bridge the previously disjointed areas of solid-state and ultrafast fiber photonics, and provides spatiotemporal selfmode locking in both fiber and solid-state oscillators.

In this article, we demonstrate that graded dissipation, provided by loss and gain transverse profiling in a GRIN fiber, allows for obtaining DKLM in a fiber laser, operating in either the anomalous or the normal dispersion regime. The parameters and stability of the resulting spatiotemporal DSs are investigated both analytically and numerically. The problem of the self-emergence (or self-starting) of a DS, and 
TABLE I. Normalization parameters for a GRIN Yb-fiber laser with dispersion compensation, and $10 \mathrm{~nm}$ spectral bandwidth. $E_{0}$ and $P_{0}$ correspond to normalization values for energy and peak power, respectively. The length of the fiber with compensated dispersion is $3 \mathrm{~m}$. The "aperture size" defines the zero level of net gain for $|\Lambda|=$ 0.002 , and $\kappa=0.001$.

\begin{tabular}{lc}
\hline \hline Wavelength & $1.06 \mu \mathrm{m}$ \\
\hline$n_{0}$ & 1.48 \\
$n_{1}$ & $0.02 \mathrm{~cm}^{-2}$ \\
$n_{2}$ & $3.5 \times 10^{-16} \mathrm{~cm}^{2} / \mathrm{W}$ \\
$\beta_{2}$ & $30 \mathrm{fs}^{2} / \mathrm{cm}^{2}$ \\
$w_{0}$ & $83 \mu \mathrm{m}$ \\
$L_{d}$ & $6 \mathrm{~cm}$ \\
$T_{0}=\sqrt{\left|\beta_{2}\right| L_{d}}$ & $230 \mathrm{fs}$ \\
$E_{0}=w_{0}^{2} T_{0} /\left(k_{0} L_{d} n_{2}\right)$ & $128 \mathrm{~nJ}$ \\
$P_{0}=w_{0}^{2} /\left(k_{0} L_{d} n_{2}\right)$ & $550 \mathrm{~kW}$ \\
$\tau$ & 0.4 \\
"Aperture size" $d=w_{0}^{2} \sqrt{|\Lambda| / \kappa}$ & $118 \mu \mathrm{m}$ \\
\hline \hline
\end{tabular}

the interdisciplinary outlook for fiber DKLM oscillators are also discussed. Besides the direct high impact in the field of photonics, such devices could be considered as a tool for the "metaphoric" modeling of spatiotemporal pattern and condensate formation in dissipative nonlinear systems, in particular, in a weakly dissipative BEC $[4,30]$.

\section{APPROACHES AND METHODS}

As it was pointed out in Refs. [5,16,17,31], the GrossPitaevskii equation, which is the well-known "workhorse" for trapped BEC modeling [32], is also a well-working approximation for describing pulse propagation in both single and multimode fibers. This equation allows for using the variational approximation (VA) for obtaining a solitonlike solution in a nondissipative GRIN fiber [7-9,19]. The generating Lagrangian $L$ for the Gross-Pitaevskii equation with a quasiparabolic trapping potential can be written as [9]

$$
\begin{aligned}
L= & \frac{i}{2}\left[a^{*} \partial_{z} a-a \partial_{z} a^{*}\right]+\frac{1}{2}\left(\left|\partial_{x} a\right|^{2}+\left|\partial_{y} a\right|^{2}\right) \\
& +\frac{\epsilon}{2}\left|\partial_{t} a\right|^{2}-\frac{v}{2}|a|^{4}-\frac{s}{2}\left(x^{2}+y^{2}\right)^{2 m}|a|^{2},
\end{aligned}
$$

where $a(z, t, x, y)$ is a slowly varying spatiotemporal field profile ( $a^{*}$ corresponds to a complex conjugated value), $z$ is a longitudinal propagation coordinate, normalized to the diffraction length $L_{d}=\beta_{0} w_{0}^{2}$, and the transverse spatial coordinates $(x, y)$ are normalized to $w_{0}=1 / \sqrt[4]{2 k_{0}\left|n_{1}\right| \beta_{0}}$. Here, $\beta_{0}=n_{0}\left(\omega_{0}\right) k_{0}$ is a propagation constant, $k_{0}=\omega_{0} / c$ is a wave number, and $n_{0}\left(\omega_{0}\right)$ is a refractive index at the carrier frequency $\omega_{0} . n_{1}$ defines a "curvature" of the transverse refractive index variation, so that $s=+1$ or $s=-1$ correspond, respectively, to an anti- or guiding GRIN fiber with an $m$-degree parabolic profile ( $m \geqslant 1$ is an integer) [33]. The group-velocity and the group-velocity dispersion (GVD) parameters are $\beta_{1}=(d \beta / d \omega)_{\omega=\omega_{0}}$ and $\beta_{2}=\left(d^{2} \beta / d \omega^{2}\right)_{\omega=\omega_{0}}$, respectively $\left[\beta=n_{0}(\omega) \omega / c\right]$. The last parameter defines the local time $t$ normalization to $T_{0}=\sqrt{\left|\beta_{2}\right| L_{d}}$, so that $\epsilon=+1$ or $\epsilon=-1$ corresponds to the anomalous or normal GVD case, respectively. The instantaneous local field intensity $|a|^{2}$ is normalized to $k_{0} n_{2} L_{d}$ ( $n_{2}$ is a nonlinear refractive index, defining SPM), so that $v=+1$ or $v=-1$ corresponds to a self-focusing or defocusing nonlinearity, respectively (see Table I).

The pulse-width scale $\left(T_{0}=230 \mathrm{fs}\right)$ and the DS width obtained from calculations (see below), jointly with the value of the cavity group-delay dispersion ( $9000 \mathrm{fs}^{2}$, see Table I), suggest neglecting the contribution of higher-order dispersion terms in Eq. (1). However, such a contribution can be important, when the DS width approaches $100 \mathrm{fs}$, and the dispersion compensation technique leads to a net GVD that approaches zero [34].

One has to note that the used notions can vary for different physical models. For instance, the DS energy, phase, and local time coordinate for an optical system correspond to the number of bosons (mass), momentum (wave number), and transverse spatial coordinate, respectively, in the case of a BEC. Thus, the condensed-matter analog of a DS is the formation of a BEC phase for $\epsilon=1$ (anomalous GVD in optics or traditional kinetic energy of bosons in BEC). The interpretation of the $\epsilon=-1$ case (normal GVD in optics) for BEC is less straightforward (nevertheless, see Ref. [35] and the classical analog such as the Talbot effect [36]).

The dissipative generalization of the Gross-Pitaevskii model based on Eq. (1) consists in the addition of a "force" $Q$ term in the Euler-Lagrange equations, in agreement with the Kantorovitch's method [37],

$$
\begin{array}{r}
\frac{\delta \int_{-\infty}^{\infty} L d t}{\delta \mathrm{f}}-\frac{d}{d z} \frac{\delta \int_{-\infty}^{\infty} L d t}{\delta \mathrm{f}}=2 \operatorname{Re} \int_{-\infty}^{\infty} Q \frac{\delta a}{\delta \mathrm{f}}, \\
Q=-i \Lambda a+i \tau \partial_{t, t} a-i \kappa\left(x^{2}+y^{2}\right)^{2 l} a,
\end{array}
$$

where $\Lambda$ is the difference between loss on the fiber axis and saturated gain (e.g., a boson exchange rate between a "basin" and a condensate for BEC [38,39]). This parameter depends upon the DS energy $\int_{-\infty}^{\infty}\left|a\left(z, x, y, t^{\prime}\right)\right|^{2} d t^{\prime}$, and it could contribute to the soliton dynamics and stability [20]. A "noninstantaneous" nonlinearity results, e.g., from gain saturation in a laser, and it can be described in the simplest form as [40]

$$
-\Lambda=\frac{g_{0}}{1+\frac{1}{E_{s}} \int_{-\infty}^{\infty}\left|a\left(z, x, y, t^{\prime}\right)\right|^{2} d t^{\prime}}-\ell,
$$

where $g_{0}$ is the unsaturated gain, $E_{s}$ is the gain saturation energy, and $\ell$ is the loss coefficient on the fiber axis.

Spectral dissipation is described by the $\tau$ parameter, which is inversely proportional to the squared bandwidth of a spectral filter (e.g., a gain medium). This parameter corresponds to, for instance, the growth of escape velocity from BEC with the boson kinetic energy.

The $\kappa$ parameter defines the growth of loss along the radial coordinate. Such a parameter is determined by graded gain and loss doping, or by the leaking loss in a fiber laser, following an $l$-degree parabolic profile ( $l \geqslant 1$ is an integer).

Equations (1) and (2) are the generating functions for the dissipative generalization of the Gross-Pitaevskii equation, which is proposed as a model for DKLM in both fiber and 
(a) highly nonlinear crystal

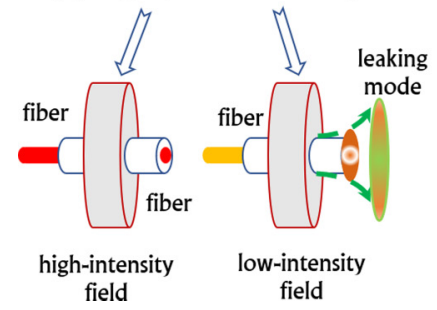

(b) tapered fiber

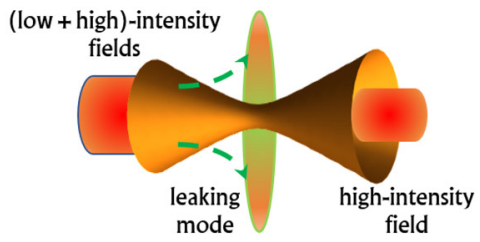

(c) fiber with graded loss or gain

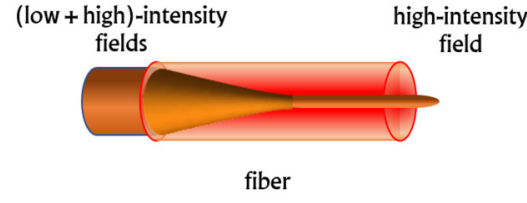

FIG. 1. Possible realizations of DKLM in an MMF laser: (a) Disjointed structure with a free beam evolving in a highly nonlinear crystal, leading to enhanced beam overlap with the subsequent fiber for a high-intensity field; (b) tapered fiber with reduced leaking mode losses for a high-intensity field; (c) MMF or photonic-crystal-fiber (PCF) structure with graded losses, providing a loss decrease due to switching to a "self-focused" high-intensity mode. The common principle is a loss decrease with intensity.

solid-state lasers,

$$
\begin{aligned}
i \frac{\partial a}{\partial z}= & \frac{1}{2}\left(\frac{\partial^{2} a}{\partial x^{2}}+\frac{\partial^{2} a}{\partial y^{2}}\right)+\frac{\epsilon}{2} \frac{\partial^{2} a}{\partial t^{2}}+\frac{s}{2}\left(x^{2}+y^{2}\right)^{2 m} a \\
& +\nu|a|^{2} a-i \Lambda a+i \tau \frac{\partial^{2} a}{\partial t^{2}}-i \kappa\left(x^{2}+y^{2}\right)^{2 l} a .
\end{aligned}
$$

Under the assumption of cylindrical symmetry and zero vorticity, the transverse Laplacian in Eq. (4) reads as $\left[\partial^{2} / \partial r^{2}+(1 / r) \partial / \partial r\right]$, where $r$ is a radial coordinate. The calculations in this work are made under these assumptions [see Eqs. (5), (6), and (10) below].

Figure 1 demonstrates the proposed implementation of the general principle of DKLM in a fiber laser. The point is that the growth of effective gain with intensity in a fiber laser with graded loss is similar to the action of a soft or hard aperture in a solid-state Kerr-lens mode-locked laser [27], which provides mode locking resulting in DS generation.

Figure 1(a) demonstrates the scheme with a bulk nonlinear crystal integrated into a fiber. In a crystal, there is no mode confinement, so that diffraction increases the mode mismatch between "in"- and "out"-fiber sections. The nonlinear beam squeezing induced by Kerr nonlinearity in a crystal would provide a better mode matching for higher intensities and thereby lower leaking losses. In the scheme of Fig. 1(b), the leaking losses are induced by the tapered fiber section. As it was previously anticipated, such losses can be reduced by mode squeezing, caused by the self-focusing effect in a fiber.

Figure 1(c) shows, in our view, the most promising approach to DKLM in a fiber laser, which preserves the fiber integrity, and uses the graded refractive index and dissipation (loss or gain) to provide loss decrease for a more intense field, owing to the Kerr nonlinearity of the fiber. Such a section can be easily integrated into an all-fiber laser, based on an MMF with graded dissipative (active or loss) doping, or a PCF.

\section{FUNDAMENTAL MODE DS}

Below we will consider the fundamental mode DS [41] in the framework of the VA for either axially symmetric parabolic $(m=1)$ or superparabolic $(m=2)$ trapping potentials, which approximate the refractive index profile of usual GRIN fibers [33]. At the same time, the last case is closer to a fiber with a steplike refractive index profile, and weaker intermode overlapping. Moreover, we will assume that the transverse profile of dissipation "traces" the refractive index one, i.e., $l=m$.

\section{A. Fundamental mode DS for $m=l=1$}

In this case, the reduced Lagrangian $\tilde{L}$ required for the VA, specifically

$$
\tilde{L}=\int_{-\infty}^{\infty} \int_{0}^{\infty} \int_{0}^{2 \pi} r L d t d r d \vartheta
$$

is calculated by using a trial function, which corresponds to a solitonlike Gaussian mode,

$$
\begin{aligned}
a(z, t, r)= & \alpha(z) \operatorname{sech}\left(\frac{t}{T(z)}\right) \\
& \times \exp \left[i\left(\phi(z)+\psi(z) t^{2}+\theta(z) r^{2}\right)-\frac{r^{2}}{2 \rho(z)^{2}}\right] .
\end{aligned}
$$

Here, the $\mathrm{f}=(\alpha, T, \phi, \psi, \theta, \rho)$ parameters describe the $z$ dependent DS amplitude, duration, phase delay ( $\partial_{z} \phi$ could be interpreted as a DS wave number), chirp, wave-front curvature (spatial chirp), and beam size, respectively. The variation $\delta^{*} / \delta \mathrm{f}$ in Eq. (2) is performed over these parameters. $r=$ $\sqrt{x^{2}+y^{2}}, \vartheta$, and $t$ are the radial and azimuthal cylindrical coordinates, and local time, respectively. In this notation, $x=r \cos (\vartheta)$ and $y=r \sin (\vartheta)$. The $(x, y, t)$ coordinates can be reinterpreted as transverse spatial coordinates $(x, y, z)$, and the "evolutionary" coordinate $z$ in Eqs. (1) and (4) can be reinterpreted as a time coordinate $T$ for BEC if $\epsilon=1$.

The VA demonstrates the existence of a nondissipative soliton solution of Eqs. (1) and (4) for $\epsilon=1, v=1, s=$ -1 , i.e., without taking into account dissipative factors in Eqs. (2) and (4) (that is, $\Lambda=\kappa=\tau=0$ ) [8,9]. However, the Vakhitov-Kolokolov (VK) stability criterion $d E / d q>0$ [3], where $E=\pi \alpha^{2} T \rho^{2}$ is a soliton energy, and $q=\partial_{z} \phi$ is its wave number, suggests that such a soliton is stable only above a certain intensity threshold of $\alpha^{2}>3.46$ (or $\approx 27 \mathrm{GW} / \mathrm{cm}^{2}$ in our normalizations), which is hardly realistic for a fiber laser. Moreover, numerical simulations (see the Appendix) show a collapselike behavior of such a soliton, in the presence of spatiotemporal chirp perturbations.

On the other hand, it is natural to conjecture that "selfemergence" could exist whenever the multidimensional soliton is supported by dissipation. Indeed, the VA-based analysis demonstrates the existence of chirp-free solitons with nonzero wave-front curvature in the anomalous GVD regime $(\epsilon=1)$, 


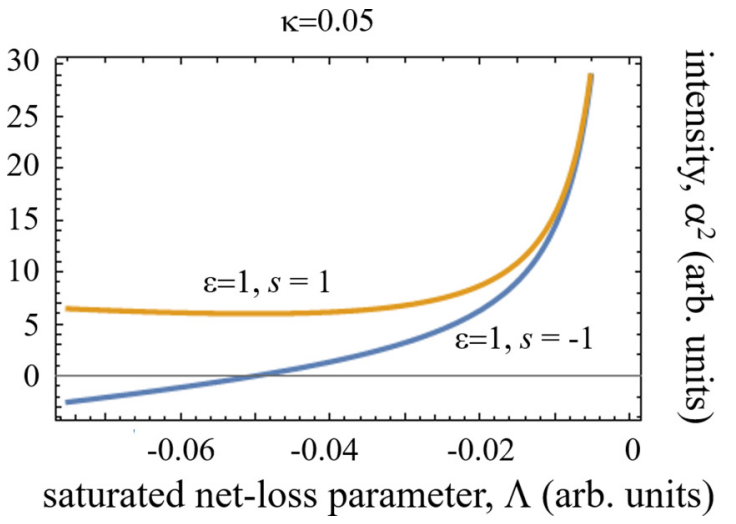

FIG. 2. Dependence of pulse intensity $\alpha^{2}$ on the saturated netloss parameter $\Lambda$ for a chirp-free DS (i.e., with no spectral dissipation, $\tau=0)$, and anomalous GVD $(\epsilon=1)$ : (i) Antiguiding ( $s=1$, orange curve), and (ii) guiding ( $s=-1$, blue curve) GRIN fiber.

for both guiding $(s=-1)$ and antiguiding $(s=1)$ refractive index grading. Spatially graded dissipation supports spatial confinement in both cases, under the condition of the absence of spectral dissipation [i.e., with $\tau=0$ in Eq. (4)]. The DS parameters are

$$
\alpha^{2}=\frac{3\left(\Lambda^{4}-s \Lambda^{2}-\kappa^{2}\right)}{\kappa \Lambda \nu}, \quad T^{2}=\frac{2 \epsilon}{\nu \alpha^{2}}, \quad \rho^{2}=-\frac{\Lambda}{\kappa}, \quad \theta=\frac{\Lambda}{2} .
$$

Equations (7) demonstrate that the spatial structure of this type of DS is formed by the spatially graded confinement of dissipation, with an effective aperture size $\chi=\sqrt{|\Lambda| / \kappa}$. The DS duration and intensity are inversely related to each other, as it occurs with $(1+1)$ D nonlinear Schrödinger solitons. However, at variance with the latter, the DS intensity is determined by both refractive and dissipative guiding properties of a fiber. The "deconfinement" $\left(\alpha^{2} \rightarrow 0\right)$, which occurs for $|\Lambda| \rightarrow \kappa$ and $s=-1$ (see Fig. 2), means that the DS may also exist when the peak power is reasonably low (depending upon the net-loss $\Lambda$ variation). This situation is of interest for the self-starting of fiber laser mode locking.

The VK stability criterion $d E / d q>0$ [3], where $q=$ $\partial_{z} \phi=\left(\kappa^{2}+5 s \Lambda^{2}-5 \Lambda^{4}\right) /(4 \kappa \Lambda)$, demonstrates the local stability ("attracting basin") of such a DS for saturated gain parameter values $\Lambda \in\left\{0,-\sqrt{\sqrt{9+20 \kappa^{2}}-3 / 10}\right\}$ (see Fig. 3). However, our numerical analysis (see the Appendix) shows that this "attracting basin" is extremely narrow, due to the crucial contribution to DS collapse of both temporal and spatial chirp perturbations [42]. As a result, such a DS cannot be self-emerging.

Therefore, one may conjecture that a self-emerging spatiotemporal DS should necessarily be chirped (i.e., $\psi \neq 0$ ), due to the contribution of spectral dissipation, i.e., one has to set $\tau \neq 0$ in Eq. (4). The solution, in this case, corresponds to

$$
\begin{aligned}
\psi & =\frac{120 \tau}{\pi^{2} T^{2}\left(15 \epsilon+\sqrt{15} \sqrt{15 \epsilon^{2}+128 \tau^{2}}\right)}, \\
\alpha^{2} & =\frac{3+3 s \rho^{4}-3 \kappa^{2} \rho^{8}}{v \rho^{2}},
\end{aligned}
$$

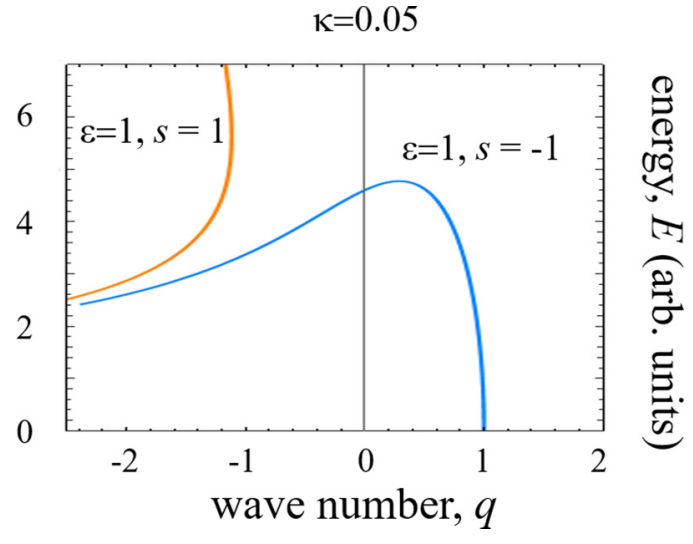

FIG. 3. Dependence of the energy $E$ on the wave number $q$ for a chirp-free DS (i.e., with $\tau=0)$, anomalous GVD $(\epsilon=1)$, and antiguiding ( $s=1$, orange curve) or guiding ( $s=-1$, blue curve) GRIN fiber.

$$
\begin{aligned}
\theta & =-\kappa \rho^{2} / 2, \\
T^{2} & =\frac{2 \rho^{2}\left(\epsilon+\frac{80 \tau^{2}\left[15 \epsilon\left(\pi^{2}-9\right)+\left(\pi^{2}+3\right) \sqrt{225 \epsilon^{2}+1920 \tau^{2}}\right]}{\pi^{2}\left(15 \epsilon+\sqrt{225 \epsilon^{2}+1920 \tau^{2}}\right)^{2}}\right)}{3+3 s \rho^{4}-3 \kappa^{2} \rho^{8}} .
\end{aligned}
$$

The resulting equation for the beam-area parameter $\rho^{2}$,

$$
\begin{aligned}
\frac{\left(12+\pi^{2}\right) \tau}{3 \pi^{2}}+\Lambda T^{2}= & \frac{120 \epsilon \tau}{\pi^{2}\left(\sqrt{15} \sqrt{15 \epsilon^{2}+128 \tau^{2}}+15 \epsilon\right)} \\
& +\frac{2880 \tau^{3}}{\pi^{2}\left(\sqrt{15} \sqrt{15 \epsilon^{2}+128 \tau^{2}}+15 \epsilon\right)^{2}} \\
& -\frac{\kappa \rho^{2} T^{2}}{2},
\end{aligned}
$$

has a unique physical solution (see the Appendix). The corresponding dependence of the beam area parameter $\rho^{2}$ on the graded dissipation parameter $\kappa$ is shown in Fig. 4, whereas the relationship between DS temporal width and intensity is shown in Fig. 5, for the case of normal GVD. A DS is positively chirped in both the anomalous GVD and in the normal GVD regimes $(\psi>0)$, and it exhibits a negative wave-front curvature $(\theta<0)$.

In the case of the DS described by Eqs. (7), a decrease of the effective aperture size $\chi=\sqrt{|\Lambda| / \kappa}$ results in beam squeezing, accompanied by a growth of the peak power (see Fig. 2), whereas for the DS expressed by Eqs. (8) and (9), decreasing $\chi$ (by increasing $\kappa$ ) widens the beam (see Fig. 4 ). This means that different mechanisms of DS formation are in place for the two cases. In the former case, spatial and temporal mechanisms are only related via the peak power of the DS, which is defined by the effective beam size. On the other hand, in the latter case one deals with an interplay between nondissipative and dissipative mechanisms, as it occurs in mode-locked lasers operating in either the anomalous GVD or the normal GVD regime. Dissipative factors may stabilize a soliton. However, their overdoing leads to soliton degradation.

As it was pointed in Ref. [17], the fundamental mode approximation is quite precise "on average" for the description of soliton dynamics in a GRIN fiber. Thus, our primary intention here is to inspect the "attraction basin" of DSs, on 

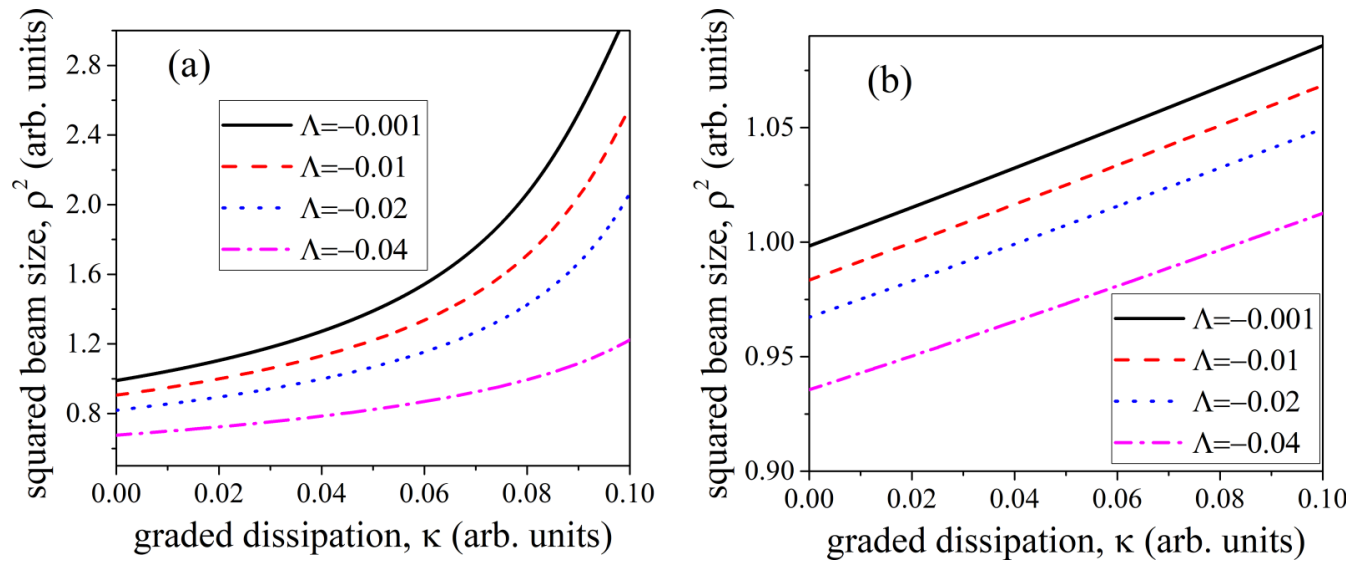

FIG. 4. Dependence of beam area $\propto \rho^{2}$ on the graded dissipation parameter $\kappa$, for different values of the saturated net-loss parameter $\Lambda$. Here, the spectral dissipation parameter is (a) $\tau=0.1$ or (b) $\tau=1 ; \epsilon=-1$ (normal GVD), $s=-1$.

the basis of numerical simulations of the system of ordinary differential equations describing the DS parameter evolution, as obtained in the framework of the VA (see the Appendix).

Figure 6 demonstrates the evolution of the DS temporal duration and peak power for two different "seed" amplitudes $\alpha(0)$, for propagation in the normal GVD regime (for other parameters, please refer to the caption of Fig. 6). Other initial conditions formally correspond to a nondissipative soliton solution of Eqs. (1) and (2) for $\Lambda=\kappa=\tau=0: T(0)=\sqrt{2|\epsilon| / v} / \alpha(0), \rho(0)=$ $\left[v \alpha(0)^{2}-\sqrt{v^{2} \alpha(0)^{4}-36 s}\right] / 6 s, \psi(0)=0, \theta(0)=0$ (see the Appendix). As can be seen in Fig. 6, the pulse width and intensity both converge to a fixed value after a certain propagation distance. The existence of such convergent solutions underlies the finding of stability borders, as marked by curves and scatter symbols in Fig. 7.

As a matter of fact, numerical simulations demonstrate the existence of a broad attracting basin of stable $(2+1)$ dimensional DSs. This indicates the possibility of DS self-

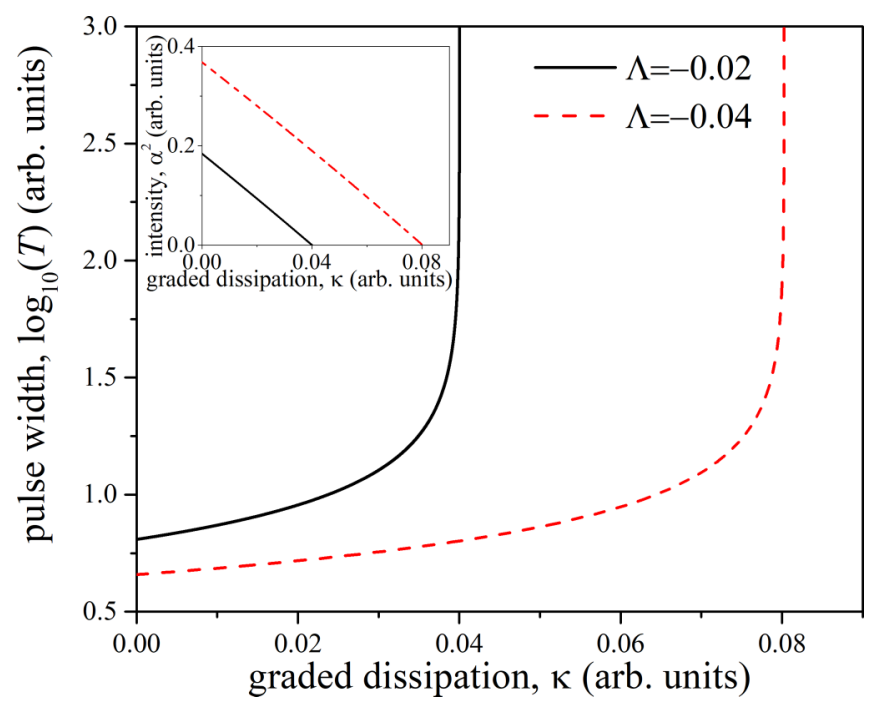

FIG. 5. Dependence of DS temporal width and intensity (inset) on the grading dissipation parameter $\kappa$, for two values of the saturated net loss $\Lambda$. Here, $\tau=1$, and $\epsilon=-1$ (normal GVD). emergence from an arbitrary initial Gaussian small signal with a given amplitude $\alpha_{0}$. Figure 7 (curves and scatter points) shows that spectral dissipation enhances DS stability, and broadens the "attraction basin," i.e., the DKLM capability, which is not possible to achieve in the absence of spectral dissipation, that is, for $\tau=0$. The growth of the graded dissipation index $\kappa$ reduces the stability region (Fig. 7), and increases the DS duration (Fig. 5). As one can see from Fig. 7, the DS stability regions are broader for the case of normal GVD, and the sensitivity to spectral dissipation is weaker in this case.

In Fig. 6(b), the solid curve shows the formation of an initial spike (with a three orders-of-magnitude increase of intensity with respect to the asymptotic solution obtained at a large distance). The presence of such "blow-up" dynamics in the generation of a DS can prevent its formation. Our calculations demonstrate that this phenomenon is more pronounced in the normal GVD regime, as it was reported for a DKLM solid-state oscillator [43]. The rescaling of the fiber laser parameters from Table I to those of a DKLM solid-state oscillator [44] demonstrates that the theory presented here bridges both fiber and solid-state waveguide mode-locked lasers, and explains the DS energy scalability at high levels of SPM, which is inherent in a fiber laser and artificially created in a DKLM solid-state oscillator $[28,29]$.

\section{B. Fundamental mode DS for $m=l=2$}

Let us consider in this section a spatial confinement potential which is closer to a step-profiled refractive index, which increases intermodal dispersion and thus decreases nonlinear mode coupling. In this case, our ansatz function for the VA has a super-Gaussian profile,

$$
\begin{aligned}
a(z, t, r)= & \alpha(z) \operatorname{sech}\left(\frac{t}{T(z)}\right) \\
& \times \exp \left[i\left(\phi(z)+\psi(z) t^{2}+\theta(z) r^{4}\right)-\frac{r^{4}}{4 \rho(z)^{4}}\right] .
\end{aligned}
$$

The nontrivial results for a chirp-free solution $(\psi=0)$ are the following: (i) The stability threshold obtained from the VK 

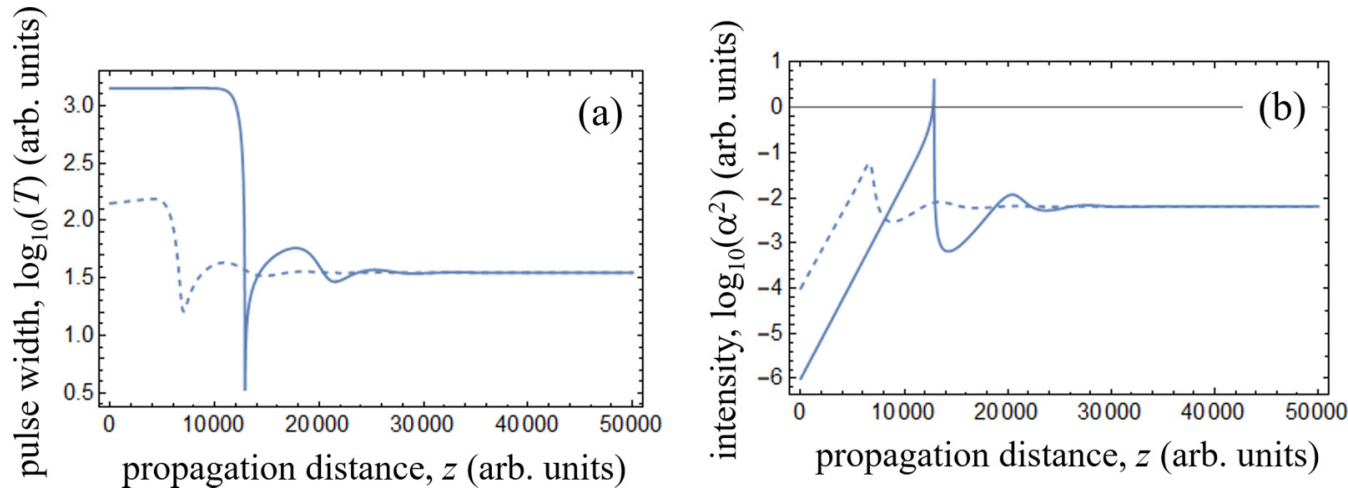

FIG. 6. (a) Dynamics of the evolution of the DS temporal width $T$ and (b) intensity $\alpha^{2}$ for an initial weak "seed" $\alpha(0)=0.001$ (solid curve) or $\alpha(0)=0.01$ (dashed curve). Here, $\tau=0.5, \chi=1.22, \kappa=0.001, \epsilon=-1$ (normal GVD), $s=-1$, and $v=1$.

stability criterion is almost than twofold lower $\left(\alpha^{2}>2.08\right.$, or $\approx 16 \mathrm{GW} / \mathrm{cm}^{2}$ in our normalization) in a nondissipative case (i.e., $\Lambda=\kappa=\tau=0$ ) than that obtained for a paraboliclike refractive index profile, and (ii) there is no DS in the absence of spectral dissipation (i.e., with $\Lambda \neq 0, \kappa \neq 0$, and $\tau=0$ ).

In the presence of spectral dissipation $(\tau \neq 0)$, the VA based on the ansatz (10) results in the following expressions for the DS parameters [45],

$$
\begin{aligned}
\alpha^{2} & =\frac{3}{4 \nu \sqrt{\pi} \rho^{2}}\left[8+\sqrt{\pi} \rho^{6}\left(4 \sqrt{2} s-3 \sqrt{\pi} \kappa^{2} \rho^{6}\right)\right], \\
\theta & =-\frac{1}{8} \sqrt{\frac{\pi}{2}} \kappa \rho^{2}, \\
T^{2} & =\frac{2 \sqrt{2 \pi} \rho^{2}\left(\frac{80 \tau^{2}\left(\left(3+\pi^{2}\right) \sqrt{225 \epsilon^{2}+1920 \tau^{2}}+15\left(\pi^{2}-9\right) \epsilon\right)}{\pi^{2}\left(\sqrt{225 \epsilon^{2}+1920 \tau^{2}}+15 \epsilon\right)^{2}}+6 \epsilon\right)}{9\left(-3 \pi \kappa^{2} \rho^{12}+4 \sqrt{2 \pi} \rho^{6} s+8\right)},
\end{aligned}
$$

and $\psi$ corresponds to Eq. (8). The resulting equation for the beam area parameter $\rho^{2}$ reads as

$$
\begin{aligned}
& \frac{2880 \tau^{3} T^{2}}{\pi^{2}\left(\sqrt{15} \sqrt{128 \tau^{2}+15 \epsilon^{2}}+15 \epsilon\right)^{2}}-\kappa \rho^{4} T^{2} \\
& +\frac{120 \tau \epsilon}{\pi^{2}\left(\sqrt{15} \sqrt{128 \tau^{2}+15 \epsilon^{2}}+15 \epsilon\right)}=\frac{\left(12+\pi^{2}\right) \tau}{3 \pi^{2}}+\Lambda T^{2}
\end{aligned}
$$

and has a sole physical solution.

As it could be expected, in this case the beam is spatially squeezed, when compared with the $m=l=1$ case. Here, spectral dissipation has a different influence with respect to the case of a parabolic confinement potential. First, for relatively low spectral dissipation (e.g., with $\tau=0.1$ ), for $m=$ $l=2$ the DS intensity $\alpha^{2}$ is lower (higher) in the anomalous (normal) GVD regime, respectively, when compared with the case of a parabolic potential. Moreover, the DS intensity is almost $\kappa$ independent in the normal dispersion regime. Second, the DS width for $m=l=2$ is substantially lower (in normal GVD case) than that for $m=l=1$, and it remains almost $\kappa$ independent.

The stability regions obtained from numerical simulations based on the VA (see the Appendix) are shown in Fig. 8. As one could expect, these regions are narrower in terms of the effective aperture size $\chi$, in comparison with the case of a parabolic potential. Nevertheless, here the stability regions are less sensitive to spectral dissipation.

\section{SPATIOTEMPORAL MULTIMODE DYNAMICS}

Multimode spatiotemporal beam dynamics beyond the fundamental-mode approximation and the DS stability boarders presented in Fig. 7 can be grasped by means of direct numerical simulations of the dissipative Gross-Pitaevskii equation (4). We performed a series of numerical simulations of Eq. (4) by using the finite-element method, implemented by the COMSOL MULTIPHYSICS software. These simulations demonstrate the self-starting of DS generation (or DS as a "global attractor") for both anomalous and normal GVD regimes.

In the case of $\chi \rightarrow 1$, there is a strong $Q$ switching on the initial stage of mode locking [see Fig. 6(b) and Ref. [43]]. Above the stability boarders presented in Fig. 7, that is, in the instability regions of the DS solutions, we observed that higher-order spatial modes develop (see Figs. 9 and 10). As it was pointed out previously, multimode dynamics provokes a multipulse generation, and leads to a complex spatiotemporal behavior [4,21,31,46,47]. Figures 9 and 10 illustrate the scenario of a transition to DS splitting and multiple pulse generation through the excitation of higher-order spatial modes. The physics of the underlying process can be commented on in the following way. An excitation of higher-order spatial modes causes energy leaking into them, so that single pulse generation becomes energetically unfavorable. As a result, a system tends to relax to a state characterized by either the generation of multiple pulses, or a continuous-wave emission.

Several approaches can be envisaged, in order to suppress these instabilities. Dynamical gain saturation [see Eq. (3)] is a key factor for DS stabilization [48], including the spatiotemporal case $[5,20,21]$. In the framework of the approach proposed in this work, such a saturation amounts to a "selfadjusting" of the effective aperture size $\chi$, which permits us to control mode coupling, depending on DS energy. This effect could provide a "passive negative feedback" mechanism, thus enhancing the DS stability: Further study is required to assess its feasibility. Such investigations will have an interdisciplinary impact, as they may shed light on the 

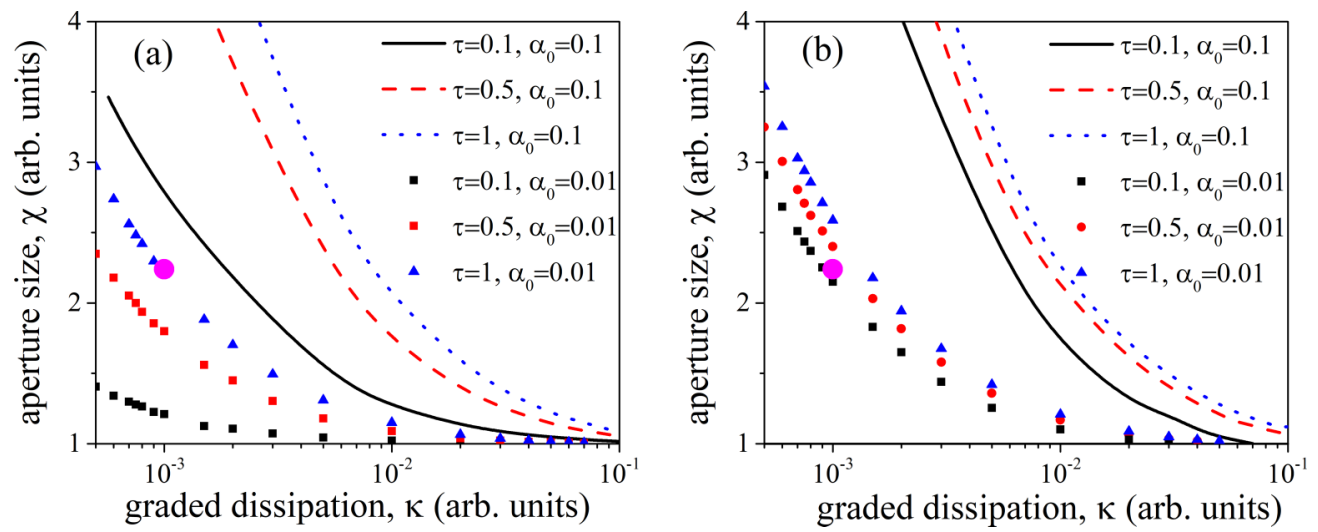

FIG. 7. Dependence of the upper boundaries of DS stability in either the anomalous GVD [(a) $\epsilon=1]$ or the normal GVD [(b) $\epsilon=-1]$ dispersion regime, respectively, vs the dimensionless "aperture size" $\chi=\sqrt{|\Lambda| / \kappa}$, for different spectral dissipation parameters $\tau$. The curve and scatter point plots trace the stability boundaries for different initial field amplitude $\left(\alpha_{0}\right)$ values. The large solid magenta circles correspond to the parameters of multimode simulations presented in Figs. 9 and 10.

role of noninstantaneous nonlinearities in the control of wave turbulence in out-of-equilibrium nonlinear systems [4,49].

Another approach could be based on the manipulation of the confining or trapping complex potential, aimed at the control of the multimode dynamics in an MMF. In particular, by using step-index-like profiled fibers (see above) one could decouple the propagation of higher-order modes from the DS mode. This approach is, in some sense, equivalent to soft and hard aperture approaches in the KLM of a solid-state oscillator $[27,50]$. The decoupling of nondissipative and dissipative potentials [e.g., $m \neq l$ in Eq. (4) that is realized in a tapered fiber [5]] could attain this goal as well. Both approaches could be realized in either PCF or standard multicore fiber platforms, in particular, composed of different materials [4,21,51,52].

Lastly, DS management by the periodic modulation of laser parameters can affect DS stability substantially [53]. In particular, dissipative and nondissipative sections of the laser can be physically disjointed, as it takes place in typical

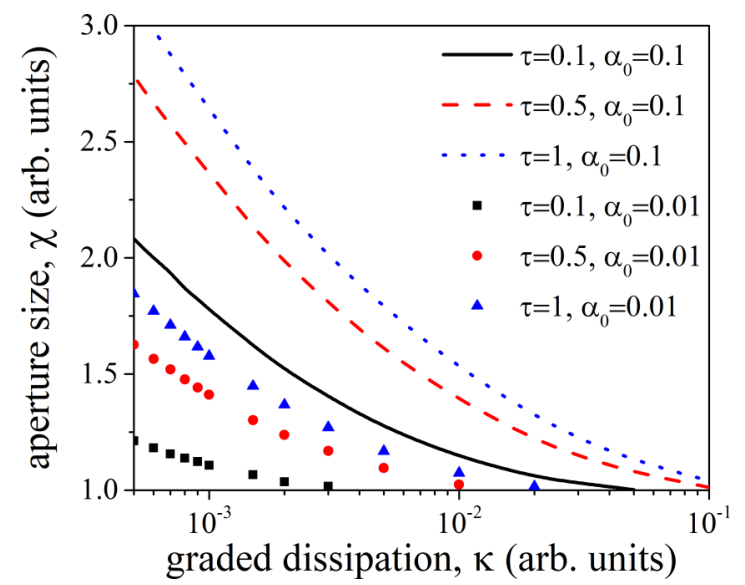

FIG. 8. Dependence of the upper boundaries of the DS stability for a super-Gaussian spatial confinement profile (i.e., for $m=l=$ $2)$ in the anomalous GVD dispersion regime $(\epsilon=1)$ upon the dimensionless "aperture size" $\chi=\sqrt[4]{|\Lambda| / \kappa}$, for different spectral dissipation parameters $\tau$. The curve and scatter point plots trace the stability boundaries for different initial field amplitude $\left(\alpha_{0}\right)$ values. fiber lasers. The confinement potentials can have a periodic localization, as well [see Figs. 1(a) and 1(b)]. The modulation of GVD and diffraction, as well as confinement potentials, can be realized by a spatially periodic modulation of the refractive index, fiber structure, and doping. For instance, the $z$ coordinate corresponds to an oscillator transition number (or a time $T$ for BEC), and the $t$ coordinate corresponds to a local time, which is associated with the coordinate frame comoving with a soliton. Thereby, the last is "embedded" into an oscillator period. For BEC, the temporal coordinate corresponds to a third transverse spatial one. Thus, the longitudinal refractive index modulation is equivalent to typical active phase mode locking forming a new confinement potential in the $t$ dimension (i.e., 3D spatial confinement in BEC). As was pointed out in Ref. [54], such a phase modulation in the $t$ dimension is sufficient for the 1D confinement of a DS, even

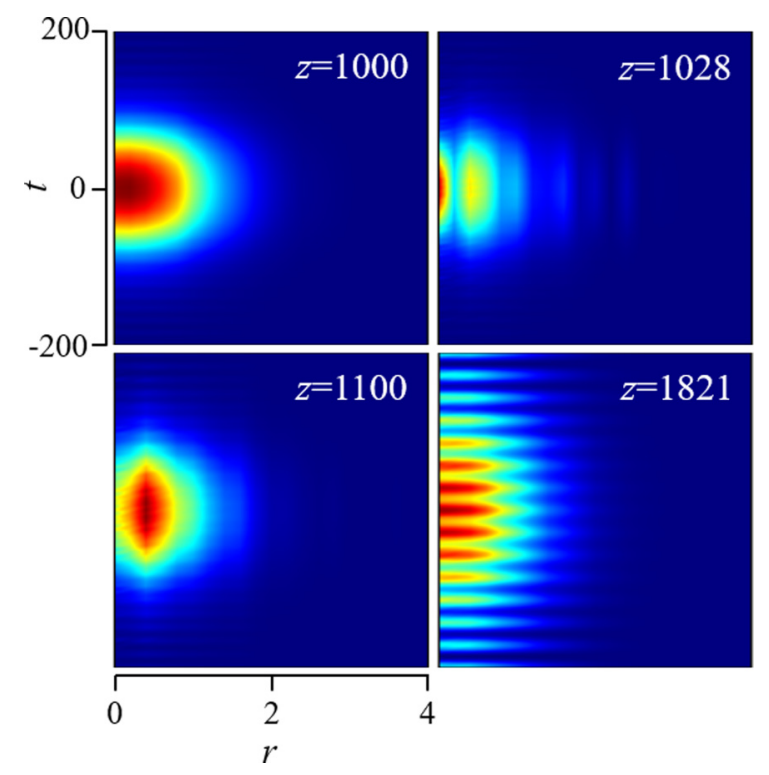

FIG. 9. Contour plots of the DS intensity illustrate the multimode spatiotemporal dynamics for $\epsilon=1$ (anomalous GVD), $\chi=2.24$, $\tau=0.1$, and $\kappa=0.001$. The coordinates correspond to Eq. (4). 


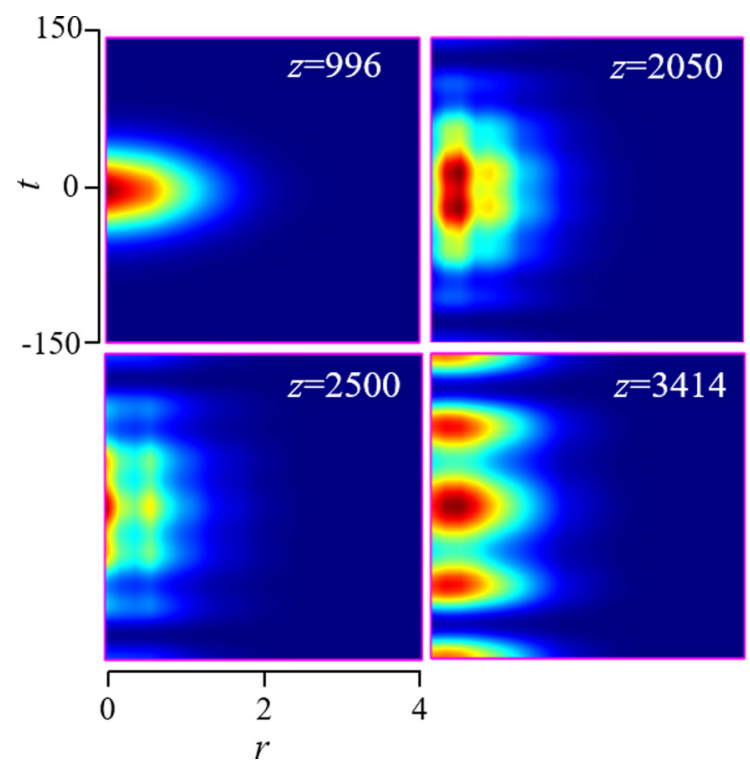

FIG. 10. Same as in Fig. 9, but for $\epsilon=-1$ (normal GVD).

in the case of an extremely low "modulation depth," when SPM, spectral dissipation, and gain are present. The possible realization of such full-dimensional confinement in an MMF laser needs further studies.

\section{CONCLUSION}

In brief summary, our study demonstrates that exploiting spatially structured dissipative effects could lead to a desirable and feasible breakthrough in mastering energy-scalable and well-controllable spatiotemporal dissipative solitons in a fiber self-mode-locked laser. The background approach is to utilize a spatially profiled dissipation (e.g., the excitation of leaking radiation, by using waveguide arrays, multicore, or multimode fibers) with the aim of stabilizing the DS, and even providing a robust mechanism of self-starting spatiotemporal mode locking. In fact, our concept is closely related to the spacetime spectral duality $[55,56]$ involved in spatiotemporal mode locking. Such a mechanism of spatiotemporal DS formation can be considered as a path to achieve energy-scalable DKLM in large-mode-area solid-state lasers, in MMF lasers, as well as in photonic lattices.

We anticipate that the nonlinear coupling of spatial modes in either graded-index or photonic-crystal fibers, supported by the presence of graded dissipation, could implement the concept of DKLM in a fiber laser in the regime of multimode self-cleaning. This would provide the means to achieve highly efficient and stable energy harvesting in an all-fiber laser, without the need for using any additional mode-locking mechanisms. In a broader context, we envisage that photonic devices could provide an efficient tool for metaphorical or analog modeling [15] of strongly localized coherent (or partially coherent) structures, which spontaneously emerge in nonlinear nonequilibrium dissipative systems. In particular, these systems represent a classical analog of a Bose-Einstein condensate in the weakly dissipative limit.

\section{ACKNOWLEDGMENTS}

This work has received funding from the European Union Horizon 2020 research and innovation program under the Marie Skłodowska-Curie Grant No. 713694 (MULTIPLY), the European Research Council Advanced Grant No. 740355 (STEMS), and the Russian Ministry of Science and Education Grant No. 14.Y26.31.0017. V.L.K. acknowledges fruitful discussions with Dr. A. Apolonskii, who inspired the concept of Kerr-lens mode locking in a fiber laser.

\section{APPENDIX}

Our analytical approach is based on a variational approximation [Eqs. (1) and (2)] to the Gross-Pitaevskii equation (4). We have taken into account dissipative factors, which, in particular, are relevant to describing multidimensional laser systems and a weakly dissipative Bose-Einstein condensate. The corresponding algebra is rather cumbersome, and here we sketch some results, which are closely connected with the main text of the article (the calculation details can be found in Refs. [57-59]).

In the nondissipative case (i.e., $\Lambda=\kappa=\tau=0$ ), the VA for the Gaussian ansatz and $m=1$ results in the following dynamical system for the evolution of the multidimensional soliton parameters,

$$
\begin{aligned}
& \psi^{\prime}(z)=\frac{\nu \alpha(z)^{2}}{\pi^{2} T(z)^{2}}-\frac{2 \epsilon}{\pi^{2} T(z)^{4}}+2 \epsilon \psi(z)^{2}, \quad \theta^{\prime}(z)=\frac{1}{6}\left(-3 s+\frac{\nu \alpha(z)^{2}}{\rho(z)^{2}}+12 \theta(z)^{2}-\frac{3}{\rho(z)^{4}}\right), \\
& \phi^{\prime}(z)=\frac{\epsilon}{3 T(z)^{2}}-\frac{7}{12} \nu \alpha(z)^{2}+\frac{1}{\rho(z)^{2}}, \quad \alpha^{\prime}(z)=\alpha(z)[\epsilon \psi(z)+2 \theta(z)], \\
& \rho^{\prime}(z)=-2 \theta(z) \rho(z), \quad T^{\prime}(z)=-2 \delta T(z) \psi(z) .
\end{aligned}
$$

This system has a unique soliton (i.e., steady-state) solution for the beam area, which is physically relevant, due to the absence of a minimal threshold on $\alpha^{2}$,

$$
T^{2}=\frac{2 \epsilon}{v \alpha^{2}}, \quad \rho^{2}=\frac{v \alpha^{2}-\sqrt{v^{2} \alpha^{4}-36 s}}{6 s}
$$

where the intensity $\alpha^{2}$ can be treated as a free parameter. 
In the nondissipative case of an $m=2$ potential and with the super-Gaussian ansatz, one has

$$
\begin{aligned}
& \psi^{\prime}(z)=\frac{\sqrt{2} \nu \alpha(z)^{2}}{\pi^{2} T(z)^{2}}-\frac{2 \epsilon}{\pi^{2} T(z)^{4}}+2 \epsilon \psi(z)^{2} \\
& \theta^{\prime}(z)=-\frac{s}{2}+\frac{\nu \alpha(z)^{2}}{6 \sqrt{2} \rho(z)^{4}}+24 \sqrt{\frac{2}{\pi}} \theta(z)^{2} \rho(z)^{2}-\frac{1}{\sqrt{2 \pi} \rho(z)^{6}} \\
& \phi^{\prime}(z)=\frac{\epsilon}{3 T(z)^{2}}-\frac{\nu \alpha(z)^{2}}{\sqrt{2}}+\frac{3-16 \theta(z)^{2} \rho(z)^{8}}{\sqrt{2 \pi} \rho(z)^{2}} \\
& \alpha^{\prime}(z)=-\alpha(z)\left(-\epsilon \psi(z)-8 \sqrt{\frac{2}{\pi}} \theta(z) \rho(z)^{2}\right) \\
& \rho^{\prime}(z)=-8 \sqrt{\frac{2}{\pi}} \theta(z) \rho(z)^{3}, \quad T^{\prime}(z)=-2 \epsilon T(z) \psi(z) .
\end{aligned}
$$

The physically relevant steady-state solution of (A3) for the beam area is

$$
\rho^{2}=-\frac{\sqrt[3]{\sqrt{2} \sqrt{s^{3}\left(1458 s-\sqrt{2} \pi \alpha^{6} v^{3}\right)}+54 s^{2}}}{3 \sqrt{2} \sqrt[6]{\pi} s}-\frac{\sqrt[6]{\pi} \alpha^{2} v}{3 \sqrt[3]{\sqrt{2} \sqrt{s^{3}\left(1458 s-\sqrt{2} \pi \alpha^{6} v^{3}\right)}+54 s^{2}}} .
$$

For the complex parabolic potential $m=l=1$ with nonzero $\Lambda, \kappa$, and $\tau$ parameters [see Eqs. (1), (2), and (4)], the evolution of the DS parameters obeys

$$
\begin{aligned}
\psi^{\prime}(z) & =\frac{3 \nu \alpha(z)^{2}-4\left(3+\pi^{2}\right) \tau \psi(z)}{3 \pi^{2} T(z)^{2}}-\frac{2 \epsilon}{\pi^{2} T(z)^{4}}+2 \epsilon \psi(z)^{2} \\
\theta^{\prime}(z) & =\frac{1}{6}\left(-3 s+\frac{\nu \alpha(z)^{2}}{\rho(z)^{2}}+12 \theta(z)^{2}-\frac{3}{\rho(z)^{4}}\right) \\
\phi^{\prime}(z) & =\frac{\epsilon}{3 T(z)^{2}}-\frac{7}{12} v \alpha(z)^{2}+\frac{1}{\rho(z)^{2}}+\frac{1}{9}\left(3+\pi^{2}\right) \tau \psi(z), \\
\alpha^{\prime}(z) & =\frac{1}{15} \alpha(z)\left(3 \pi^{2} \tau T(z)^{2} \psi(z)^{2}-\frac{5\left(12+\pi^{2}\right) \tau}{\pi^{2} T(z)^{2}}+15[-\Lambda+\epsilon \psi(z)+2 \theta(z)]\right), \\
\rho^{\prime}(z) & =-\rho(z)\left[2 \theta(z)+\kappa \rho(z)^{2}\right] \\
T^{\prime}(z) & =-2 \epsilon T(z) \psi(z)-\frac{16}{15} \pi^{2} \tau T(z)^{3} \psi(z)^{2}+\frac{8 \tau}{\pi^{2} T(z)} .
\end{aligned}
$$

These equations define the solutions (7)-(9) and underlie Figs. 2-7.

The system for the $m=l=2$ potential and the super-Gaussian ansatz (10),

$$
\begin{aligned}
& \psi^{\prime}(z)=\frac{3 \sqrt{2} \nu \alpha(z)^{2}-4\left(3+\pi^{2}\right) \tau \psi(z)}{3 \pi^{2} T(z)^{2}}-\frac{2 \epsilon}{\pi^{2} T(z)^{4}}+2 \epsilon \psi(z)^{2}, \\
& \theta^{\prime}(z)=\frac{\sqrt{\pi} \nu \alpha(z)^{2} \rho(z)^{2}+288 \theta(z)^{2} \rho(z)^{8}-6}{6 \sqrt{2 \pi} \rho(z)^{6}}-\frac{s}{2} \\
& \phi^{\prime}(z)=\frac{\epsilon}{3 T(z)^{2}}-\frac{v \alpha(z)^{2}}{\sqrt{2}}+\frac{3-16 \theta(z)^{2} \rho(z)^{8}}{\sqrt{2 \pi} \rho(z)^{2}}+\frac{1}{9}\left(3+\pi^{2}\right) \tau \psi(z), \\
& \alpha^{\prime}(z)=\frac{1}{15} \alpha(z)\left[3 \pi^{2} \tau T(z)^{2} \psi(z)^{2}-\frac{5\left(12+\pi^{2}\right) \tau}{\pi^{2} T(z)^{2}}-15\left(\Lambda-8 \sqrt{\frac{2}{\pi}} \theta(z) \rho(z)^{2}-\epsilon \psi(z)\right)\right], \\
& \rho^{\prime}(z)=-\rho(z)^{3}\left(8 \sqrt{\frac{2}{\pi}} \theta(z)+\kappa \rho(z)^{2}\right), \\
& T^{\prime}(z)=-\frac{16}{15} \pi^{2} \tau T(z)^{3} \psi(z)^{2}+\frac{8 \tau}{\pi^{2} T(z)}-2 \epsilon T(z) \psi(z),
\end{aligned}
$$

underlies Eqs. (11) and (12) and Fig. 8. 
One has to note that the solution (8) for the chirp parameter $\psi$ was obtained by using the Muller's method [60] to avoid a singularity for $\tau \rightarrow 0$. Equations (9) and (12) can be reduced to the polynomials relative to the beam area $\rho^{2}$ of fourth and sixth order, respectively $[57,58]$.

[1] M. C. Cross and P. C. Hohenberg, Rev. Mod. Phys. 65, 851 (1993).

[2] P. A. Robinson, Rev. Mod. Phys. 69, 507 (1997).

[3] B. A. Malomed, Eur. Phys. J. Spec. Top. 225, 2507 (2016).

[4] Y. V. Kartashov, G. A. Astrakharchik, B. A. Malomed, and L. Torner, Nat. Rev. Phys. 1, 185 (2019).

[5] K. Krupa, A. Tonello, A. Barthélémy, T. Mansuryan, V. Couderc, G. Millot, Ph. Grelu, D. Modotto, S. A. Babin, and S. Wabnitz, APL Photonics 4, 110901 (2019).

[6] V. N. Serkin and T. L. Belyaeva, Optik 176, 38 (2019).

[7] M. Karlsson, D. Anderson, and M. Desaix, Opt. Lett. 17, 22 (1992).

[8] Sh.-Sh. Yu, Ch.-H. Chien, Y. Lai, and J. Wang, Opt. Commun. 119, 167 (1995).

[9] S. Raghavan and G. P. Agrawal, Opt. Commun. 180, 377 (2000).

[10] O. V. Shtyrina, M. P. Fedoruk, Y. S. Kivshar, and S. K. Turitsyn, Phys. Rev. A 97, 013841 (2018).

[11] Ph. Grelu, J. M. Soto-Crespo, and N. Akhmediev, Opt. Express 13, 9352 (2005).

[12] Th. Mayteevarunyoo, B. A. Malomed, and D. V. Skryabin, Opt. Express 27, 37364 (2019).

[13] B. A. Malomed, J. Opt. Soc. Am. B 31, 2460 (2014).

[14] C.-K. Lam, B. A. Malomed, K. W. Chow, and P. K. A. Wai, Eur. Phys. J. Spec. Top. 173, 233 (2009).

[15] Editorial, Nat. Photonics 8, 1 (2014).

[16] L. G. Wright, Z. M. Ziegler, P. M. Lushnikov, Z. Zhu, M. A. Eftekhar, D. N. Christodoulides, and F. W. Wise, IEEE J. Sel. Top. Quantum Electron. 24, 5100516 (2018).

[17] W. H. Renninger and F. W. Wise, Nat. Commun. 4, 1719 (2013).

[18] R. Guenard, K. Krupa, R. Dupiol, M. Fabert, A. Bendahmane, V. Kermene, A. Desfarges-Berthelemot, J. L. Auguste, A. Tonello, A. Barthélémy, G. Millot, S. Wabnitz, and V. Couderc, Opt. Express 25, 22219 (2017).

[19] A. S. Ahsan and G. P. Agrawal, Opt. Lett. 43, 3345 (2018).

[20] T. Bhutta, J. I. Mackenzie, D. P. Shepherd, and R. J. Beach, J. Opt. Soc. Am. B 19, 1539 (2002).

[21] L. G. Wright, P. Sidorenko, H. Pourbeyram, Z. M. Ziegler, A. Isichenko, B. A. Malomed, C. R. Menyuk, D. N. Christodoulides, and F. W. Wise, Nat. Phys. 16, 565 (2020).

[22] U. Tegin, E. Kakkava, B. Rahmani, D. Psaltis, and Ch. Moser, Optica 6, 1412 (2019).

[23] Ph. Grelu and N. Akhmediev, Nat. Photonics 6, 84 (2012).

[24] M. E. Fermann, A. Galvanauskas, and G. Sucha, Ultrafast Lasers: Technology and Applications (Dekker, New York, 2003).

[25] J. I. Mackenzie, IEEE J. Sel. Top. Quantum Electron. 13, 626 (2007).

[26] H. Byun, D. Pudo, S. Frolov, A. Hanjani, J. Shmulovic, E. P. Ippen, and F. X. Kartner, IEEE Photonics Technol. Lett. 21, 763 (2009).
[27] D. E. Spence, P. N. Kean, and W. Sibbett, Opt. Lett. 16, 42 (1991).

[28] J. Zhang, J. Brons, M. Seidel, D. Bauer, D. Sutter, V. Pervak, V. Kalashnikov, Z. Wei, A. Apolonski, F. Krausz, and O. Pronin, in Advanced Solid State Lasers Conference, OSA Technical Digest (Optical Society of America, Washington, D.C., 2015), p. ATh4A.7.

[29] W. H. Renninger and F. W. Wise, Optica 1, 101 (2014).

[30] Universal Themes of Bose-Einstein Condensation, edited by N. P. Proukakis, D. W. Snoke, and P. B. Littlewood (Cambridge University Press, Cambridge, U.K., 2017).

[31] L. G. Wright, W. H. Renninger, D. N. Christodoulides, and F. W. Wise, Opt. Express 23, 3492 (2015).

[32] L. Pitaenskii and S. Stringari, Bose-Einstein Condensation and Superfluidity (Oxford University Press, Oxford, UK, 2016).

[33] A. Mafi, J. Lightwave Technol. 30, 2803 (2012).

[34] E. Sorokin, N. Tolstik, V. L. Kalashnikov, and I. T. Sorokina, Opt. Express 21, 29567 (2013).

[35] M. V. Berry, J. Phys. A: Math. Theor. 43, 415302 (2010).

[36] J. Wen and Y. Zhang, Adv. Opt. Photonics 5, 83 (2013).

[37] S. Ch. Cerda, S. B. Cavalcanti, and J. M. Hickmann, Eur. Phys. J. D 1, 313 (1998).

[38] Yu. Kagan, A. E. Muryshev, and G. V. Shlyapnikov, Phys. Rev. Lett. 81, 933 (1998).

[39] Yu. V. Bludov and V. V. Konotop, Phys. Rev. A 81, 013625 (2010).

[40] V. L. Kalashnikov, Chirped-pulse oscillators: Route to the energy-scalable femtosecond pulses, in Solid State Laser, edited by A. H. Al-Khursan (IntechOpen, Rijeka, 2012), Chap. 5.

[41] As was pointed in Ref. [17], such an approximation is effective on average, even in the case of multimode oscillations in a GRIN fiber. Moreover, the GRIN fiber model can be considered as a rough approximation for the description of PCF or photonic lattices [4,5].

[42] Note that, in the case of collapselike behavior, the following assumptions of the considered model become invalid: (i) paraxial approximation, (ii) lowest-order mode ansatz, and (iii) axial symmetry.

[43] O. Pronin, J. Brons, C. Grasse, V. Pervak, G. Boehm, M.-C. Amann, A. Apolonski, V. L. Kalashnikov, and F. Krausz, Opt. Lett. 37, 3543 (2012).

[44] J. Brons, V. Pervak, E. Fedulova, D. Bauer, D. Sutter, V. Kalashnikov, A. Apolonskiy, O. Pronin, and F. Krausz, Opt. Lett. 39, 6442 (2014).

[45] Note that the dimensionality of the $\theta$ and $\kappa$ parameters differs from that for the $m=l=1$ case.

[46] E. Ding, S. Lefrancois, J. N. Kutz, and F. W. Wise, IEEE J. Quantum Electron. 47, 597 (2011).

[47] S. Longhi and D. Janner, J. Opt. B: Quantum Semiclass. Opt. 6, S303 (2004).

[48] V. L. Kalashnikov, E. Podivilov, A. Chernykh, and A. Apolonski, Appl. Phys. B 83, 503 (2006). 
[49] J. Garnier, M. Lisak, and A. Picozzi, J. Opt. Soc. Am. B 29, 2229 (2009).

[50] Sh. Yefet and A. Pe'er, Appl. Sci. 3, 694 (2013).

[51] Y. V. Kartashov, B. A. Malomed, and L. Torner, Rev. Mod. Phys. 83, 247 (2011).

[52] A. M. Ortiz and R. L. Sáez, Multi-core optical fibers: Theory, applications and opportunities, in Selected Topics on Optical Fiber Technologies and Applications, edited by F. Xu and Ch. Mou (IntechOpen, Rijeka, 2018), Chap. 4.

[53] B. A. Malomed, Soliton Management in Periodic Systems (Springer, New York, 2006).

[54] V. L. Kalashnikov, I. G. Poloyko, and V. P. Mikhailov, Opt. Spectrosc. 84, 104 (1998).

[55] L. G. Wright, D. N. Christodoulides, and F. W. Wise, Science 358, 94 (2017).
[56] V. L. Kalashnikov and S. V. Sergeyev, Dissipative solitons in fibre lasers, in Fiber Laser, edited by M. Ch. Paul (IntechOpen, Rijeka, 2016), Chap. 9.

[57] V. L. Kalashnikov, The variational approach to modeling a spatiotemporal dissipative soliton (Gaussian potential) (Mathematica 11.3 notebook), 2020, http://info.tuwien.ac.at/kalashnikov/ Variational_Gaussian.nb.

[58] V. L. Kalashnikov, The variational approach to modeling a spatiotemporal dissipative soliton (super Gaussian potential) (Mathematica 11.3 notebook), 2020, http://info.tuwien.ac.at/ kalashnikov/Variational_superGaussian.nb.

[59] V. L. Kalashnikov and S. Wabnitz, arXiv:2004.00990.

[60] D. E. Muller, Math. Tables Other Aids Comput. 10, 208 (1956). 